\title{
DESIGN AND EVALUATION OF SELF-NANOEMULSIFYING DRUG DELIVERY SYSTEMS OF MANIDIPINE FOR ENHANCEMENT OF SOLUBILITY
}

\author{
SRIKANTH REDDY S, SURESH G* \\ Department of Pharmaceutics, Career Point University, Kota, Rajasthan, India. Email: suresh.gande@gmail.com
}

Received: 04 June 2019, Revised and Accepted: 28 June 2019

\begin{abstract}
Objective: The present work is aimed at developing liquid self-nanoemulsifying drug delivery system (liquid-SNEDDS) of manidipine.

Methods: The manidipine SNEDDS is formulated with excipients comprising Capmul MCM as oil phase, Transcutol P as surfactant, and Lutrol L 300 as cosurfactant. The prepared fifteen formulations of manidipine SNEDDS were performed for emulsification time, percentage transmittance, particle size, drug release, in vitro dissolution and stability studies. Ternary phase diagram plotted using Chemix software.

Results: The optimized manidipine liquid SNEDDS formulation (F14) subjected to drug-excipient compatibility studies by Fourier-transform infrared spectroscopy and characterized for particle size, zeta potential, scanning electron microscopy, and stability studies. The morphology of manidipine SNEDDS indicates spherical shape with uniform particle distribution. The percentage drug release from optimized formulation F14 (98.24 $\pm 5.14 \%$ ) was higher than that of pure drug $(39.17 \pm 2.98 \%)$. The stability data indicated no noticeable change in drug content, emulsifying properties, drug release, and appearance.
\end{abstract}

Conclusion: Hence, a potential SNEDDS formulation of manidipine developed with enhanced solubility, dissolution rate, and bioavailability.

Keywords: Manidipine, Hypertension, Self-nanoemulsifying drug delivery system, Solubility studies, Capmul MCM.

(C) 2019 The Authors. Published by Innovare Academic Sciences Pvt Ltd. This is an open access article under the CC BY license (http://creativecommons. org/licenses/by/4. 0/) DOI: http://dx.doi.org/10.22159/ajpcr.2019.v12i9.34394

\section{INTRODUCTION}

The Class II to Class IV drugs of biopharmaceutical classification system suffering with poor water solubility lead to lower intestinal absorption, lower bioavailability. Solubilizing poor water-soluble drugs is a major challenge in pharmaceutical research.

Lipid-based drug formulations increase the relative solubility of drugs in GI track by enhancing absorption. Self-nanoemulsifying drug delivery systems (SNEDDS) lipid-based formulations are most promising technology in drug delivery [1-3]. SNEDDS is defined as pre-concentrate containing a mixture of drug, surfactants, oil, and cosurfactant. The smaller size of SNEDDS improves drug dissolution by increasing area for drug release, absorption and by promoting lymphatic transport of the drug. SNEDDS formulation is used for increasing the solubility, oral bioavailability, and permeability of drug. It also protects the drug from hostile environment in GI track and is used for selective GI targeting drug delivery [4-8]

They have particle size ranging from nanometers to few microns. Based on particle size, they are further classified into SMEDDS and SNEDDS. SMEDDS forms microemulsions consisting of oil droplet size ranging between 100 and $200 \mathrm{~nm}$. SNEDDS contains the droplets whose size is less than $100 \mathrm{~nm}$.

Manidipine is used as an antihypertensive. Manidipine binds to voltagedependent calcium channels on smooth muscle cells and dissociates them, thus blocking the entrance of extracellular calcium into the cell hence preventing this contraction $[9,10]$. This produces vasodilation which decreases blood pressure.

The objective of present research is to design and characterize the liquid SNEDDS of manidipine. The ability of SNEDDS to improve dissolution rate is evaluated. The formulated SNEDDS was characterized for emulsification time, percentage transmittance, particle size, drug release, and thermodynamic stability.

\section{MATERIALS AND METHODS}

Materials

Manidipine is gifted by Aurobindo pharma limited, Hyderabad. Capryol PGMC and Acrysol K-150 Oleic acid, Lauroglycol, and Transcutol HP are procured from Gattefosse, Mumbai. Labrasol, Tween 20, Acconon, Lutrol L 300, Capmul CMC, Labrasol, Acconon and Lutrol L 300 were generous gift samples from BASF, Mumbai.

\section{Preparation of manidipine standard stock solution}

$10 \mathrm{mg}$ of working standard manidipine were transferred into a $10 \mathrm{ml}$ volumetric flask and were added $1 \mathrm{ml}$ of phosphate buffer $\mathrm{pH} 1.2$ till the mark to obtain a solution of $1000 \mu \mathrm{g} / \mathrm{ml}$. The solution diluted with phosphate buffer to get a solution concentration of $100 \mu \mathrm{g} / \mathrm{ml}$. From this solution, a series of aliquots was prepared for further method development.

\section{Solubility studies}

The solubility of manidipine in different oils (Acrysol K-150, Oleic acid, Capryol PGMC, Capmul MCM, and Labrafil), surfactants (Kolliphor ELP, Labrasol, Cremophor EL, Lauroglycol, Transcutol HP, and Tween 20), and cosurfactants (propylene glycol, Span 20 PEG 400, Acconon, and Lutrol L300) were examined by adding excess amount of manidipine (approximately $10 \mathrm{mg}$ ) with $2 \mathrm{ml}$ each of the above-mentioned components. The drug was transferred to 5 -ml glass vial and was mixed with individual components thoroughly for $10 \mathrm{~min}$. The vials placed in shaker bath at $37 \pm 1^{\circ} \mathrm{C}$ for $72 \mathrm{~h}$ to attain homogeneity. The samples were further centrifuged $8000 \mathrm{rpm}$ for $20-30 \mathrm{~min}$ at $4^{\circ} \mathrm{C}$. The supernatant removed and the drug concentration determined by ultraviolet (UV) at $228 \mathrm{~nm}[11]$. 
Construction of ternary phase diagrams

The phase diagram constructed base on the solubility of drug in various excipients. Various combinations of oil, surfactant, and cosurfactant were considered for construction of the same.

Surfactant and cosurfactant $\left(\mathrm{S}_{\text {mix }}\right)$ belonging to group were mixed in different weight ratio $(1: 1,2: 1$, and $3: 1)$. The $S_{\text {mix }}$ ratios are selected with increasing surfactant concentration with respect to increasing cosurfactant concentration and vice versa. Various weight ratios of oil and $S_{\text {mix }}$ ranging from $1: 9$ to $9: 1(1: 9,2: 8,3: 7,4: 6,5: 5,6: 4,7: 3,8: 2$, and 9:1) were taking glass vials.

Pseudoternary phase diagram plotted by aqueous titration method, each sample of oil and $\mathrm{S}_{\text {mix }}$ titrated with aqueous phase. The results tabulated based on transparency and ease of flow of emulsion. A pseudo-threecomponent phase diagram constructed to mark the physical state of the emulsion with oil on the first axis and surfactant on the second axis and cosurfactant on the third axis $[12,13]$

\section{Measurement of percentage transmittance}

The manidipine SNEDDS reconstituted with distilled water. The resulting emulsion observed visually for any turbidity. The percentage transmittance measured using UV spectrophotometer at $228 \mathrm{~nm}$ [14].

\section{Emulsification time}

With the use of virtual test method, a predetermined volume of mixture $(0.2 \mathrm{ml})$ diluted with $300 \mathrm{ml}$ of water at a temperature of $37^{\circ} \mathrm{C}$ using a magnetic stirrer. The tendency of emulsion formation observed [15]

\section{Development of manidipine SNEDDS formulations}

The manidipine SNEDDS formulations prepared using Capmul MCM were used as oil phase, and Transcutol P and Lutrol L 300 were used as surfactant and cosurfactant (Table 1). Manidipine (10 mg) was added to oil into glass vial and heated to $40^{\circ} \mathrm{C}$. The oily mixture mixed thoroughly with surfactant and cosurfactant. The mixture sonicated for $15 \mathrm{~min}$.

\section{Thermodynamic stability studies}

The formulations subjected to freeze cycle $\left(-20^{\circ} \mathrm{C}\right.$ for 2 days followed by $40^{\circ} \mathrm{C}$ for 2 days). The stable samples centrifuged at $3000 \mathrm{rpm}$ for $5 \mathrm{~min}$ and examined for any phase separation. The formulations with no phase separation selected for further investigation [16].

\section{Determination of drug content}

Manidipine formulation $(0.2 \mathrm{ml})$ equivalent to $10 \mathrm{mg}$ is taken in volumetric flask and made to $100 \mathrm{ml}$ with phosphate buffer $\mathrm{pH} 1.2 .1 \mathrm{ml}$ of this solution is taken $10 \mathrm{ml}$ flask made up to $10 \mathrm{ml}$ with buffer. The solution is diluted to $10 \mu \mathrm{g} / \mathrm{ml}$, absorbance measured at $\lambda_{\max } 228 \mathrm{~nm}$ against blank. The amount of drug present in $0.2 \mathrm{ml}$ of formulation determined by UV spectrophotometric method and drug concentration determined from standard graph.
$\%$ Drug content $=\frac{\text { Actual amount of drug in SNEDDS }}{\text { Theoretical amount of drug in SNEDDS }} \times 100$

In vitro dissolution studies of manidipine SNEDDS formulations The dissolution studies were undertaken with paddle method in $\mathrm{pH} 1.2$ phosphate buffer $(900 \mathrm{ml})$ of various concentrations of manidipine at $37^{\circ} \mathrm{C}$ and speed of $50 \mathrm{rpm}$. The liquid SNEDDS whose weight equivalent to $10 \mathrm{mg}$ of manidipine was filled into hard gelatin capsules, and samples were withdrawn at different time intervals of $2,5,10,15,20,25,30,45$, and $60 \mathrm{~min}$ and the amount of manidipine analyzed at $228 \mathrm{~nm}$ by UV $[17,18]$.

\section{Characterization of manidipine SNEDDS formulation}

Drug-excipient compatibility studies

The drug-excipient compatibility checked by Fourier-transform infrared spectroscopy (FTIR) method. An FTIR-8400S Spectrophotometer (Shimadzu, Japan) with total reflectance (ATR) accessory used to record the infrared spectra. Analysis of manidipine and physical mixtures of the drug with the excipients carried out with $\mathrm{KBr}$ disc. Eight scans recorded at a resolution of $4 \mathrm{~cm}^{-1}$ within frequency range of $400-4000 \mathrm{~cm}^{-1}$.

\section{Determination of droplet size}

The droplet size of manidipine SNEDDS formulations determined by photon correlation spectroscopy (Malvern Instrument UK) that measures the size range of 10 to $5000 \mathrm{~nm}$. The formulations placed in an electrophoretic cell after dilution [19].

\section{Determination of zeta potential}

The zeta potential of the diluted manidipine SNEDDS formulation measured using a zeta meter system. The SNEDDS diluted in ratio $1: 2500(\mathrm{v} / \mathrm{v})$ and mixed constantly. Zeta-potential of the resulting emulsion measured using a Zetasizer.

\section{Determination of shape and surface morphology}

Shape and surface morphology of manidipine SNEDDS scanned using scanning electron microscopy (SEM). The SNEDDS converted to emulsion form and placed on metal stubs coated with gold (HITACHI, S-3700N).

\section{Stability studies}

The manidipine SNEDDS formulations pilled in gelatine capsules. Stability studies conducted at $25^{\circ} \mathrm{C} / 60 \%$ relative humidity $(\mathrm{RH})$ and $40^{\circ} \mathrm{C} / 75 \% \mathrm{RH}$ in stability chambers (Thermolab, Mumbai, India). Samples at specified conditions withdrawn for 6 months. Drug content for each sample was analyzed by UV method [20].

\section{RESULTS AND DISCUSSION}

\section{Solubility studies}

The manidipine drug solubility is $<1 \mathrm{mg} / \mathrm{mL}$. The solubility of manidipine tested in oils phases (Acrysol K-150, Oleic acid, Capryol PGMC, Capmul MCM, and Labrafil). The maximum solubility observed

Table 1: Formulation trials of liquid manidipine self-nanoemulsifying drug delivery system

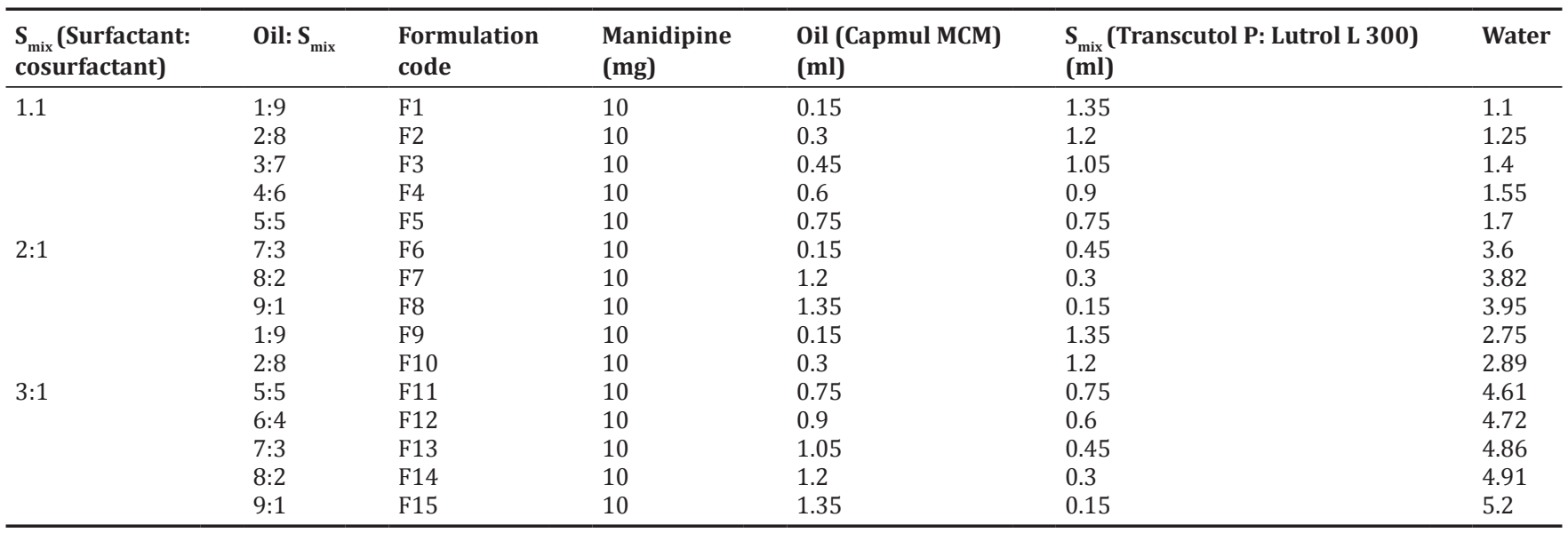


in Capmul MCM $61.84 \mathrm{mg} / \mathrm{ml}$. This was selected as oil phase for manidipine SNEDDS formulations (Table 2 and Fig. 1).

The solubility of the drug checked in various surfactants Kolliphor ELP, Labrasol, Cremophor EL, Lauroglycol, Transcutol HP, and Tween 20 (Table 3 and Fig. 2) and cosurfactants, PEG 400, Acconon, Lutrol L300, and Span 20 (Table 4 and Fig. 3). The maximum solubility found $41.22 \mathrm{mg} / \mathrm{ml}$ of Transcutol HP as a surfactant phase and $133.24 \mathrm{mg} / \mathrm{ml}$ of Lutrol L300 as a cosurfactant phase. These were chosen as surfactant and cosurfactant for SNEDDS formulation.

\section{Pseudoternary phase diagram}

From the solubility studies, Capmul MCM was chosen as oil, Transcutol $P$ as surfactant, and Lutrol L300 as cosurfactant for SNEDDS formulation. A ternary phase diagram plotted indicating that increase in concentration surfactant and cosurfactant with oil increases the self-emulsifying region. Higher efficiency of self-emulsification observed with an increase in surfactant concentration (Figs. 4-6).

\section{Visual observation}

The tendency of emulsion formation observed tested by visual observation method. This was performed by keeping the surfactant and cosurfactant ratio $\left(\mathrm{S}_{\text {mix }}\right)$ as 1:1, 2:1, and 3:1 [21]. Ratios 1:9, 2:8, 3:7, $4: 6$, and $5: 5$ of $S_{\text {mix }} 1: 1,7: 3,8: 2,9: 1,1: 9$, and $2: 8$ of $S_{\text {mix }} 2: 1$, and $5: 5,6: 4$, $7: 3,8: 2$, and 9:1 of $S_{\text {mix }} 3: 1$ exhibited rapid formation of emulsion with clear appearance within short time. Based on this data, the ratios for the formulation of SNEDDS were selected (Tables 5-7 and Fig. 7).

Table 2: Solubility of manidipine in different oils

\begin{tabular}{lll}
\hline S. No. & Oils & Solubility $(\mathbf{m g} / \mathbf{m l})$ \\
\hline 1 & Labrafil & 40.28 \\
2 & Capmul MCM & 61.84 \\
3 & Capryol PGMC & 52.26 \\
4 & Oleic acid & 10.23 \\
5 & Acrysol K 150 & 45.67 \\
\hline
\end{tabular}

Table 3: Solubility of manidipine in different surfactants

\begin{tabular}{lll}
\hline S. No. & Surfactants & Solubility $(\mathrm{mg} / \mathrm{ml})$ \\
\hline 1 & Cremophor EL & 85.11 \\
2 & Lauroglycol & 125.25 \\
3 & Kolliphor ELP & 91.21 \\
4 & Labrasol & 109.81 \\
5 & Transcutol HP & 241.22 \\
6 & Tween 20 & 35.22 \\
\hline
\end{tabular}

Table 4: Solubility of manidipine in different cosurfactants

\begin{tabular}{lll}
\hline S. No. & Cosurfactants & Solubility $(\mathrm{mg} / \mathrm{ml})$ \\
\hline 1 & Propylene glycol & 81.24 \\
2 & Acconon & 75.94 \\
3 & Lutrol L300 & 133.24 \\
4 & Propylene glycol 400 & 90.11 \\
5 & Span 20 & 61.21 \\
\hline
\end{tabular}

Table 5: Visual observation test for $S_{\text {mix }}$ (surfactant: cosurfactant) ratio 1:1

\begin{tabular}{lll}
\hline Oil: $\boldsymbol{S}_{\text {mix }}$ & Time of self-emulsification (min) & Grade \\
\hline $1: 9$ & $<2$ & III \\
$2: 8$ & $<2$ & III \\
$3: 7$ & $<2$ & III \\
$4: 6$ & $<2$ & III \\
$5: 5$ & $<2$ & III \\
$6: 4$ & $<1$ & I \\
$7: 3$ & $<1$ & I \\
$8: 2$ & $>2$ & IV \\
$9: 1$ & $>3$ & V \\
\hline
\end{tabular}

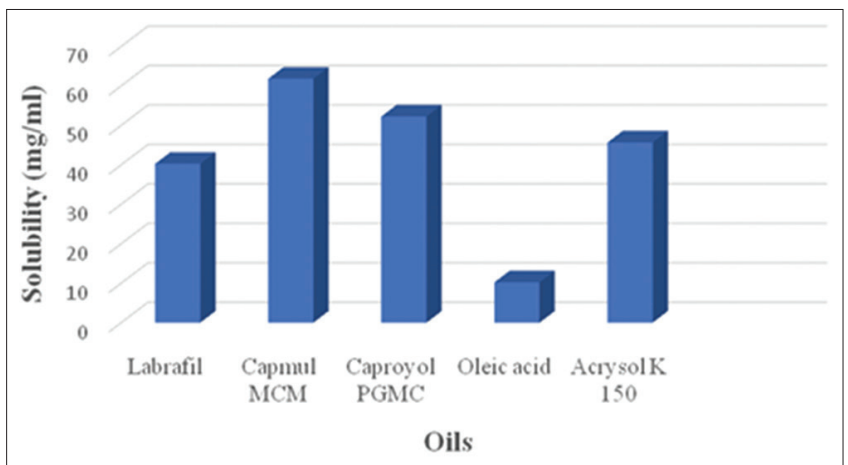

Fig. 1: Solubility studies of manidipine in oils

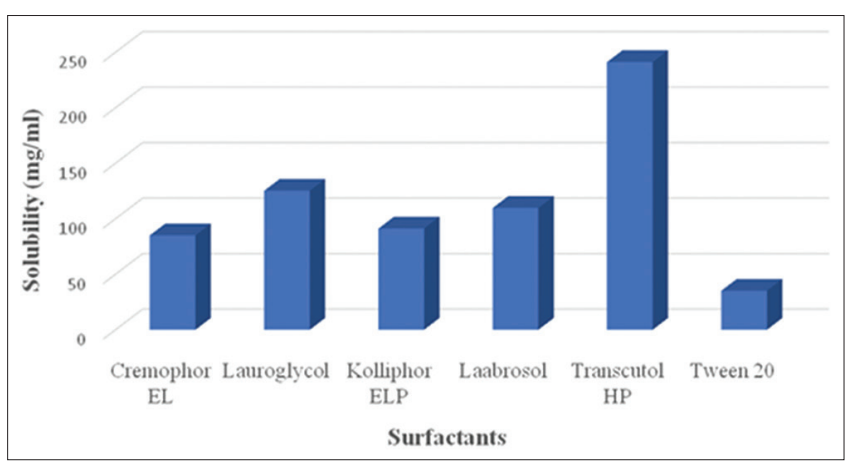

Fig. 2: Solubility studies of manidipine in surfactants

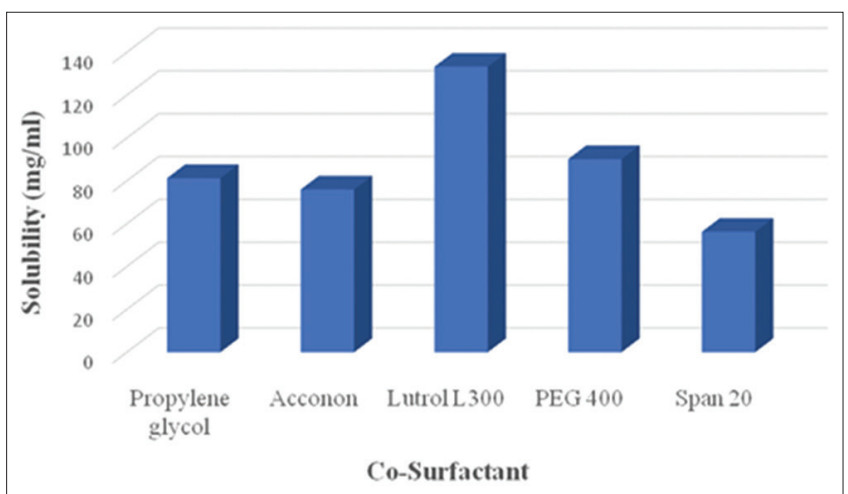

Fig. 3: Solubility studies of manidipine in cosurfactants

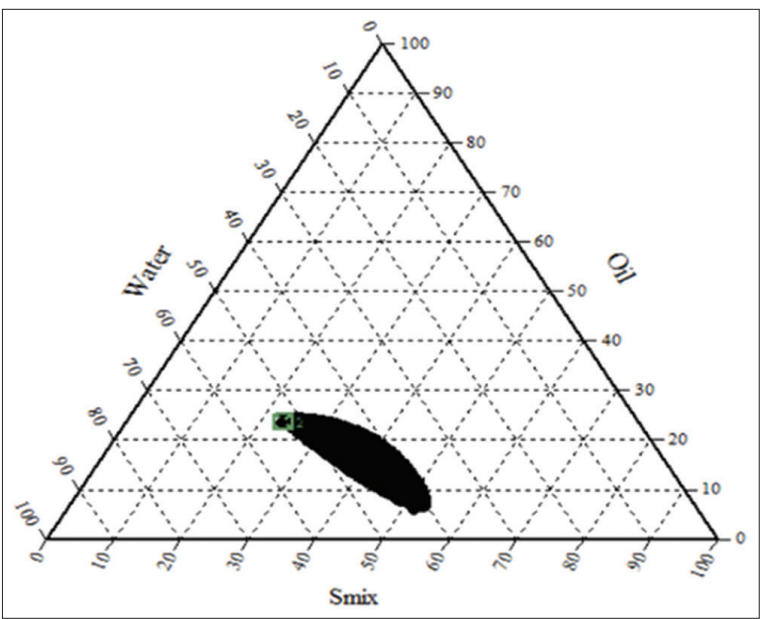

Fig. 4: Ternary phase diagram of Capmul MCM and Transcutol P and Lutrol L300 for 1:1 ratio of $S_{\text {mix }}$ 
SNEDDS of manidipine formulated using Capmul MCM (oil), Transcutol P (surfactant) and LutrolL300 (cosurfactant). All the fifteen formulations prepared were found to be clear and transparent and their complete composition analyzed (Table 1).

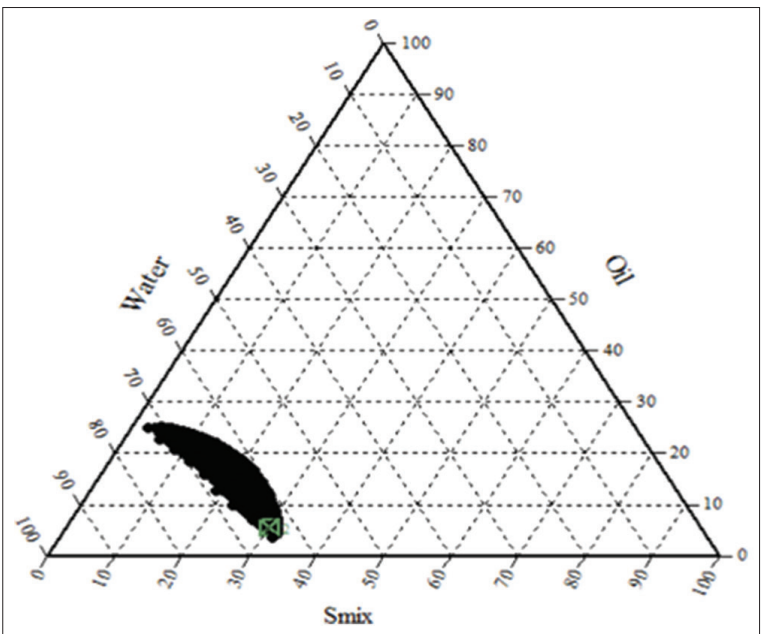

Fig. 5: Ternary phase diagram of Capmul MCM and Transcutol $P$ and Lutrol L300 for 2:1 ratio of $S_{\text {mix }}$

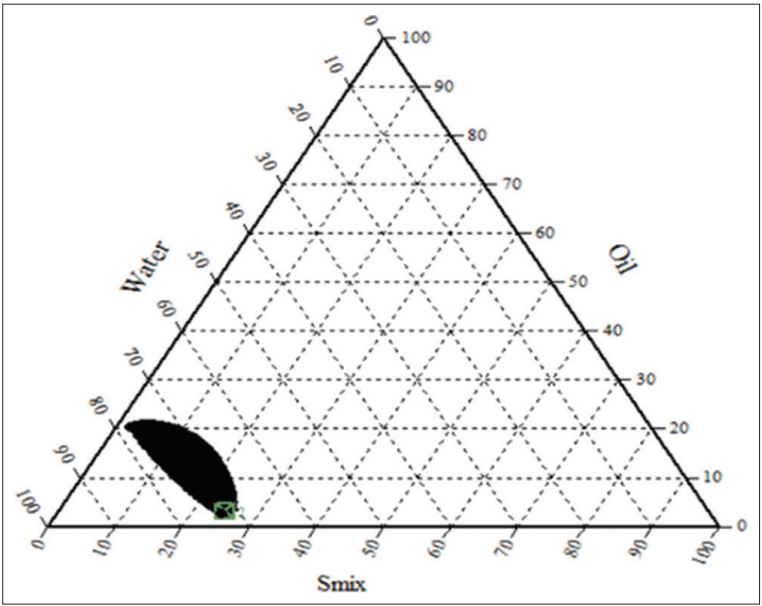

Fig. 6: Ternary phase diagram of Capmul MCM and Transcutol $P$ and Lutrol L300 for 3:1 ratio of $S_{\text {mix }}$

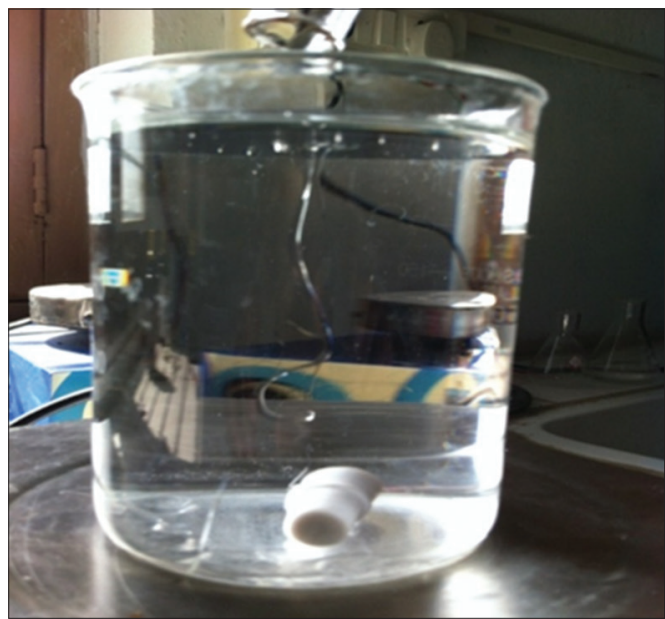

Fig. 7: Preparation of manidipine self-nanoemulsifying drug delivery system
Thermodynamic stability studies of manidipine SNEDDS

The stability studies conducted indicated no significant phase separation or effect of temperature variation on physical appearance of the formulations. No significant change observed visually even after centrifugation freeze-thaw cycles. The thermodynamically stable formulations were selected for further characterization (Table 8).

\section{$\%$ transmittance measurement}

The transmittance $(\% \mathrm{~T})$ measures the clarity and transparency of emulsions. Formulation F14 exhibited \% transmittance value $>99 \%$. Transmittance values were $<99 \%$ suggesting less clarity of emulsions (Table 9).

\section{Drug content of manidipine SNEDDS}

The drug content of the formulated manidipine SNEDDS found to be in the range of $91.19-98.96 \%$. A maximum of $98.96 \%$ was found in the formulation F14 (Table 10).

Table 6: Visual observation test for $S_{\text {mix }}$ (surfactant: cosurfactant) ratio $2: 1$

\begin{tabular}{lll}
\hline Oil: $\mathbf{S}_{\text {mix }}$ & Time of self-emulsification (min) & Grade \\
\hline $1: 9$ & $<2$ & III \\
$2: 8$ & $<2$ & III \\
$3: 7$ & $<1$ & I \\
$4: 6$ & $<1$ & I/II \\
$5: 5$ & $<1$ & I \\
$6: 4$ & $>2$ & IV \\
$7: 3$ & $<2$ & III \\
$8: 2$ & $<2$ & III \\
$9: 1$ & $<2$ & III \\
\hline
\end{tabular}

Table 7: Visual observation test for $S_{\text {mix }}$ (surfactant: cosurfactant) ratio 3:1

\begin{tabular}{lll}
\hline Oil: $\mathbf{S}_{\text {mix }}$ & Time of self-emulsification (min) & Grade \\
\hline $1: 9$ & $<1$ & I \\
$2: 8$ & $<1$ & I \\
$3: 7$ & $>2$ & IV \\
$4: 6$ & $<1$ & I \\
$5: 5$ & $<2$ & III \\
$6: 4$ & $<2$ & III \\
$7: 3$ & $<2$ & III \\
$8: 2$ & $<2$ & III \\
$9: 1$ & $<2$ & III \\
\hline
\end{tabular}

Table 8: Thermodynamic stability studies of the formulations

\begin{tabular}{|c|c|c|c|}
\hline \multirow{2}{*}{$\begin{array}{l}\text { Formulation } \\
\text { code }\end{array}$} & \multirow[t]{2}{*}{ Centrifugation } & \multicolumn{2}{|c|}{ Freeze-thaw method } \\
\hline & & $\begin{array}{l}-20^{\circ} \mathrm{C} \text { for } \\
2 \text { days }\end{array}$ & $\begin{array}{l}+40^{\circ} \mathrm{C} \text { for } \\
2 \text { days }\end{array}$ \\
\hline F1 & NPS & $\mathrm{NC}$ & $\mathrm{NC}$ \\
\hline F2 & NPS & $\mathrm{NC}$ & $\mathrm{NC}$ \\
\hline F3 & NPS & $\mathrm{NC}$ & $\mathrm{NC}$ \\
\hline F4 & NPS & $\mathrm{NC}$ & $\mathrm{NC}$ \\
\hline F5 & NPS & $\mathrm{NC}$ & $\mathrm{NC}$ \\
\hline F6 & NPS & $\mathrm{NC}$ & $\mathrm{NC}$ \\
\hline F7 & NPS & $\mathrm{NC}$ & $\mathrm{NC}$ \\
\hline F8 & NPS & $\mathrm{NC}$ & $\mathrm{NC}$ \\
\hline F9 & NPS & $\mathrm{NC}$ & $\mathrm{NC}$ \\
\hline F10 & NPS & $\mathrm{NC}$ & $\mathrm{NC}$ \\
\hline F11 & NPS & $\mathrm{NC}$ & $\mathrm{NC}$ \\
\hline F12 & NPS & $\mathrm{NC}$ & $\mathrm{NC}$ \\
\hline F13 & NPS & $\mathrm{NC}$ & $\mathrm{NC}$ \\
\hline F14 & NPS & $\mathrm{NC}$ & $\mathrm{NC}$ \\
\hline F15 & NPS & $\mathrm{NC}$ & $\mathrm{NC}$ \\
\hline
\end{tabular}

\#NPS-No phase separation. \#\#NC-No change 
Table 9: \% Transmittance of different formulations

\begin{tabular}{lll}
\hline $\begin{array}{l}\text { Formulation } \\
\text { code }\end{array}$ & $\begin{array}{l}\text { Visual } \\
\text { observation }\end{array}$ & $\begin{array}{l}\text { Percentage } \\
\text { transmittance }\end{array}$ \\
\hline F1 & Turbid & 75.19 \\
F2 & Slightly clear & 77.07 \\
F3 & Slightly clear & 78.92 \\
F4 & Turbid & 76.92 \\
F5 & Slightly clear & 79.11 \\
F6 & Slightly clear & 80.21 \\
F7 & Transparent & 85.04 \\
F8 & Transparent & 91.77 \\
F9 & Slightly clear & 84.68 \\
F10 & Slightly clear & 78.53 \\
F11 & Slightly clear & 81.24 \\
F12 & Transparent & 92.37 \\
F13 & Transparent & 94.37 \\
F14 & Transparent & 99.21 \\
F15 & Transparent & 96.25 \\
\hline
\end{tabular}

Table 10: \% drug content for various formulations of manidipine self-nanoemulsifying drug delivery system

\begin{tabular}{ll}
\hline Formulation code & Percentage drug content \\
\hline F1 & 92.37 \\
F2 & 91.19 \\
F3 & 92.93 \\
F4 & 93.99 \\
F5 & 94.74 \\
F6 & 93.66 \\
F7 & 94.67 \\
F8 & 95.37 \\
F9 & 94.64 \\
F10 & 93.44 \\
F11 & 95.93 \\
F12 & 94.79 \\
F13 & 97.99 \\
F14 & 98.96 \\
F15 & 94.19 \\
\hline
\end{tabular}

\section{In vitro dissolution studies of manidipine SNEDDS}

The drug dissolution studies indicate that the drug release from F14 is higher than that of other fourteen formulations and the pure drug (Figs. 8-10).

\section{Characterization of manidipine SNEDDS}

Drug-excipient interactions by FTIR spectroscopy

The FTIR spectra of pure drug manidipine exhibited bands at $3026.41 \mathrm{~cm}^{-1}$ due to $\mathrm{N}-\mathrm{H}$ stretch, at $1640 \mathrm{~cm}^{-1}$ for $\mathrm{C}=0$ stretching, and at $1226.77 \mathrm{~cm}^{-1}$ for aromatic amine group C-N stretching. The spectra also showed bands at $1288.49 \mathrm{~cm}^{-1}$ for C-N bending. The FTIR spectrum of SNEDDS containing manidipine exhibited characteristic bands consistent with the molecular structure of manidipine such as bands at $3095.49 \mathrm{~cm}^{-1}$ for $\mathrm{N}-\mathrm{H}$ stretch, at $1678.13 \mathrm{~cm}^{-1}$ for $\mathrm{C}=0$ stretching, and at $1226.77 \mathrm{~cm}^{-1}$ for aromatic amine group C-N stretching. The data indicate no interaction between the drug and excipients used in the formulation (Figs. 11-13).

\section{Particle size analysis of manidipine SNEDDS}

The droplet size and polydispersity values of manidipine are analyzed. The particle size of the optimized manidipine SNEDDS formulation (F14) was found to be $22.4 \mathrm{~nm}$ and Z-average $23.3 \mathrm{~nm}$. The results indicate that all the particles were in the nanometer range. The polydispersity index of manidipine SNEDDS optimized formulation (F14) was 0.313.

PDI determines the uniformity of particle diameter, and hence, it is useful to know the size distribution of nanoemulsion, which enhances good particle size distribution (Fig. 14).

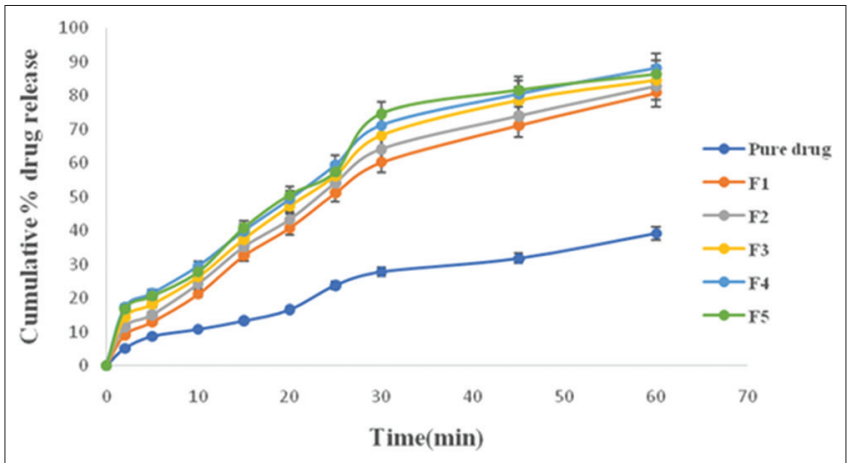

Fig. 8: Dissolution profiles of manidipine pure drug and formulations (F1 to F5)

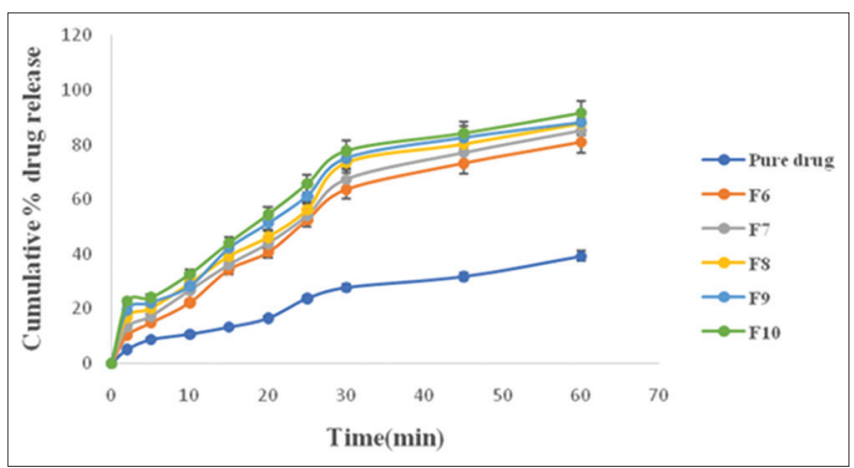

Fig. 9: Dissolution profiles of manidipine pure drug and formulations (F6 to F10)

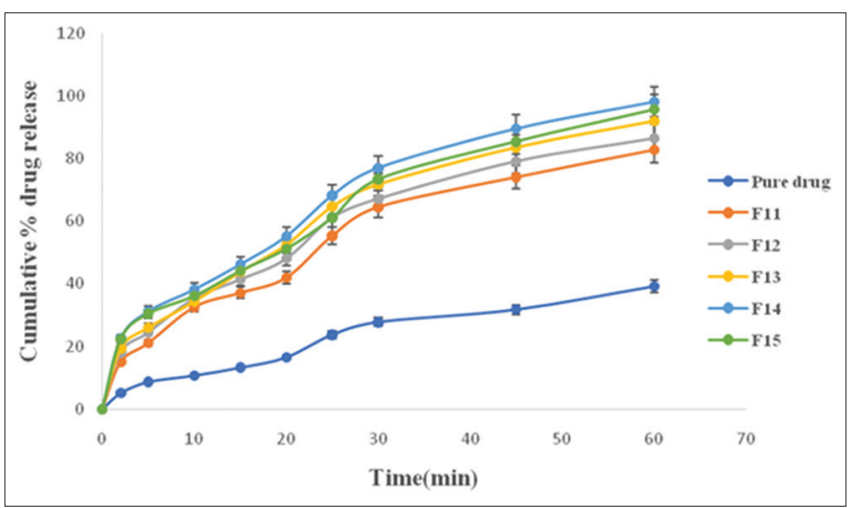

Fig. 10: Dissolution profiles of manidipine pure drug and formulations (F11 to F15)

Zeta potential of manidipine SNEDDS

The zeta potential of the optimized SNEDDS formulation was found to be $-5.1 \mathrm{mV}$ which complies with the requirement of the zeta potential for particle stability (Fig. 15).

SEM for manidipine SNEDDS

SEM evaluated the morphology of SNEDDS formulation. The data showed a spherical shape for the optimized SNEDDS (F14) formulation with uniform and relatively narrow particle distribution. Spherical particles could be uptaken easier than disfigured ones. Therefore, it is speculated that the mean particle size that was obtained using the laser diffraction method belongs to the agglomerated SNEDDS particles (Fig. 16).

\section{Stability studies}

The stability of optimized manidipine SNEDDS formulation studied for 6 months. The formulation was packed in hard gelatin capsules for the 


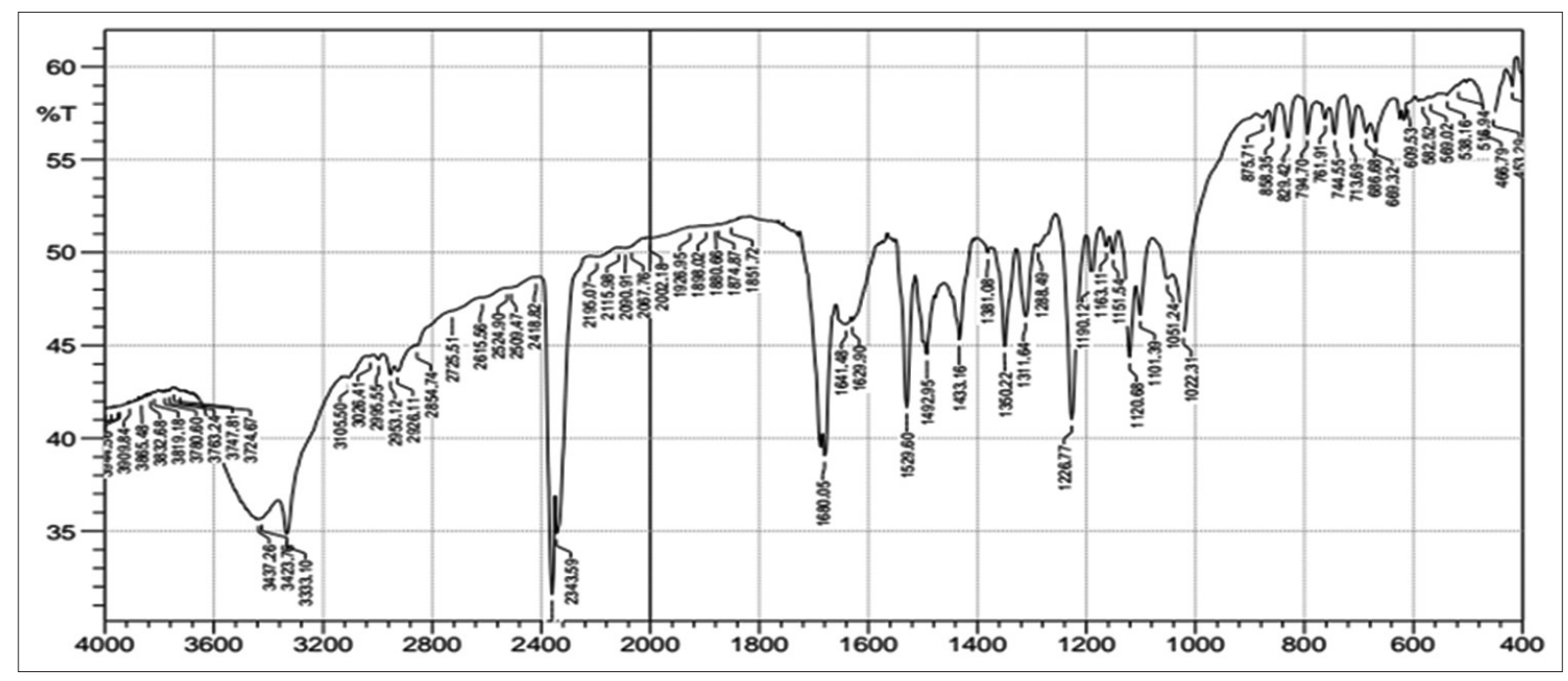

Fig. 11: Fourier-transform infrared spectroscopy spectrum of manidipine pure drug

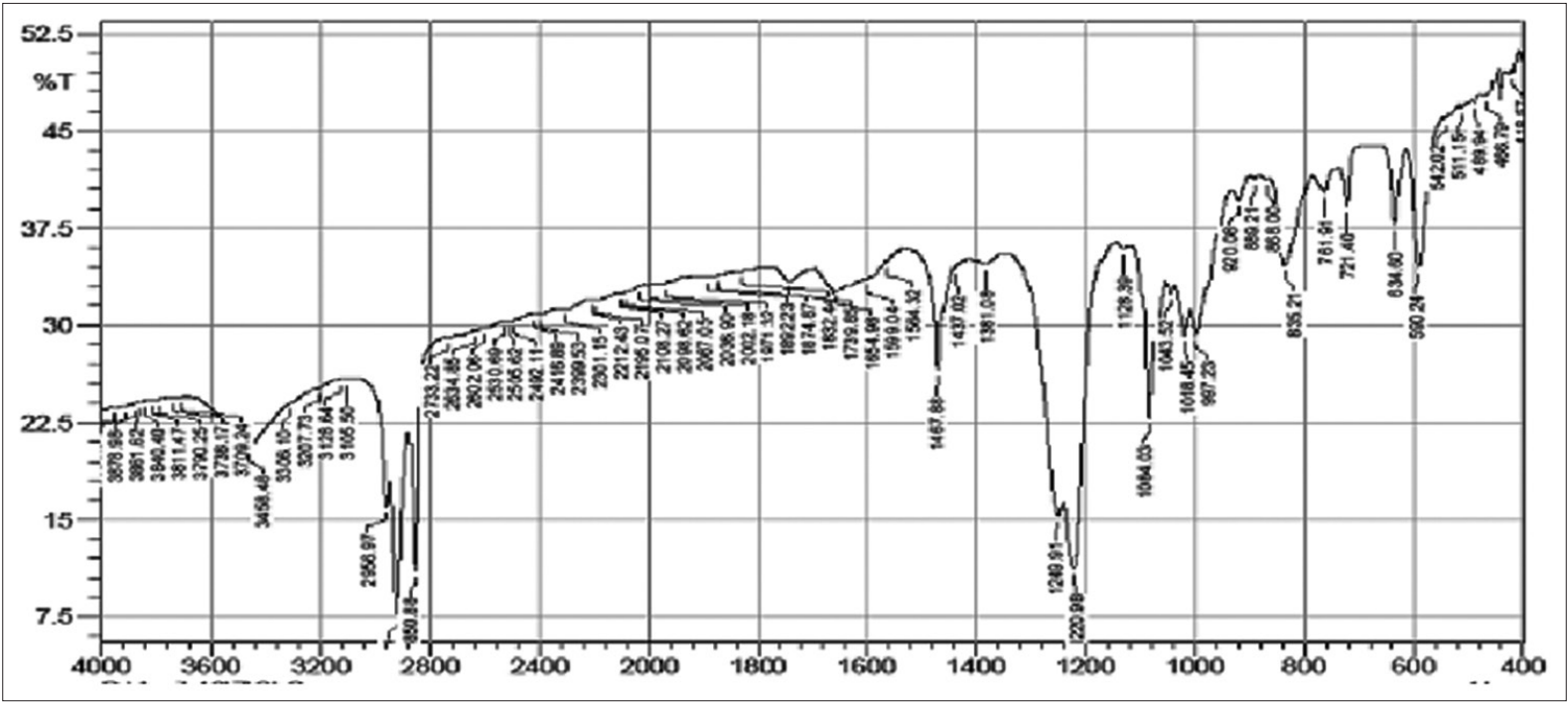

Fig. 12: Fourier-transform infrared spectroscopy spectrum of manidipine physical mixture

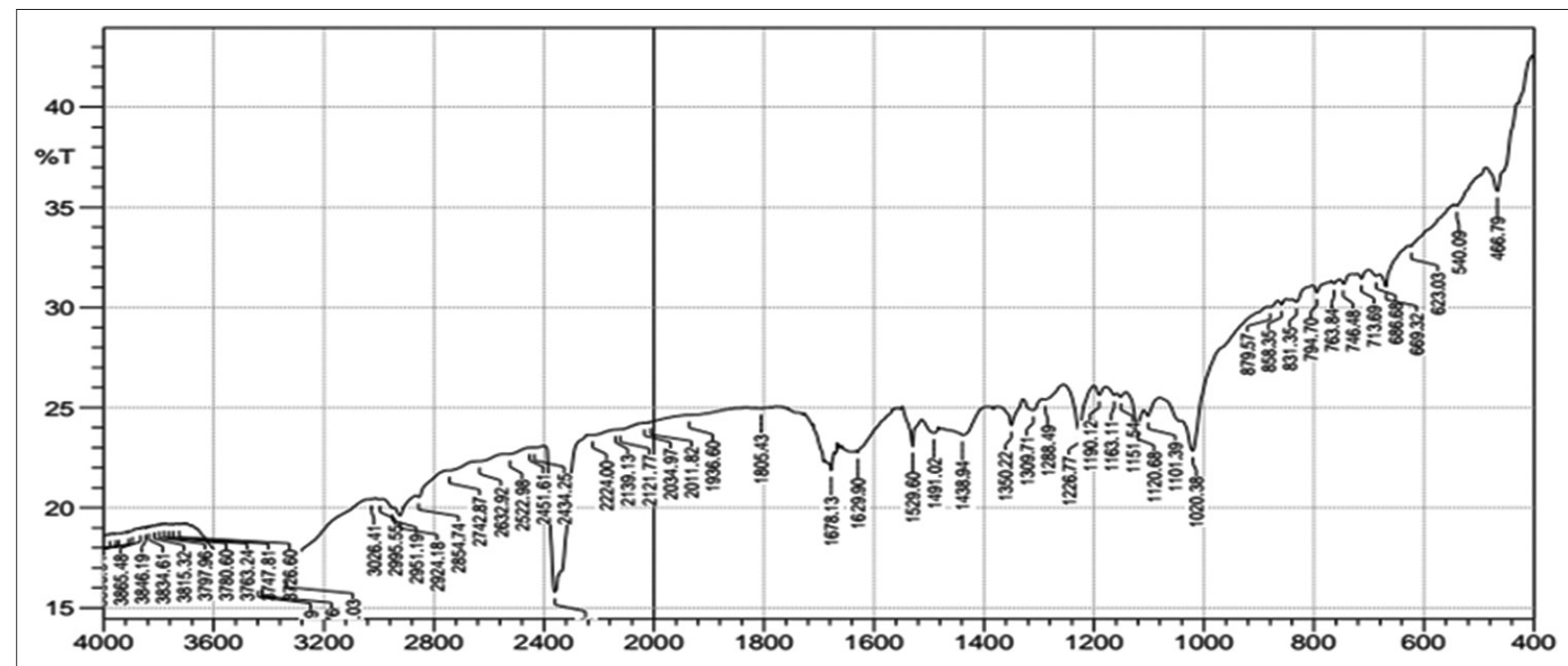

Fig. 13: Fourier-transform infrared spectroscopy spectrum of formulation F14 of manidipine self-nanoemulsifying drug delivery system 


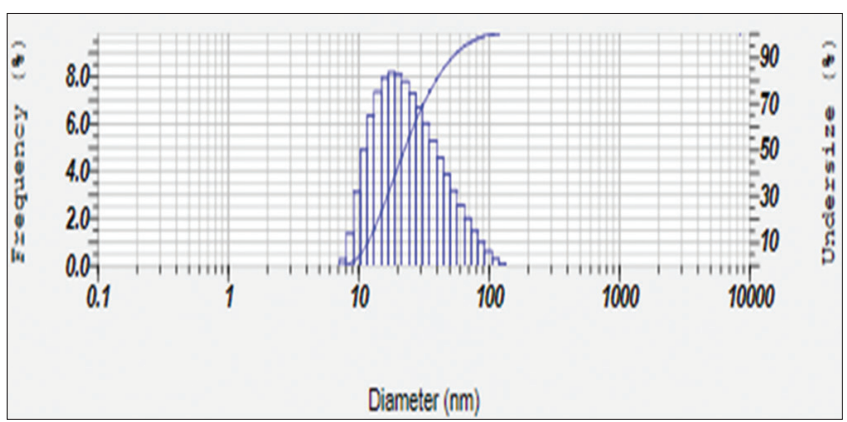

Fig. 14: Particle size analysis of manidipine self-nanoemulsifying drug delivery system formulation F14

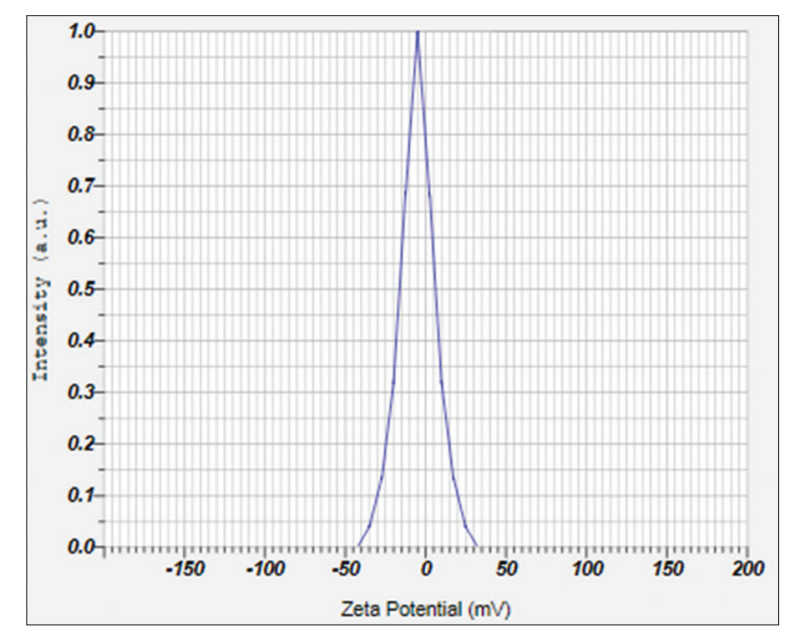

Fig. 15: Zeta potential of the manidipine self-nanoemulsifying drug delivery system optimized formulation F14
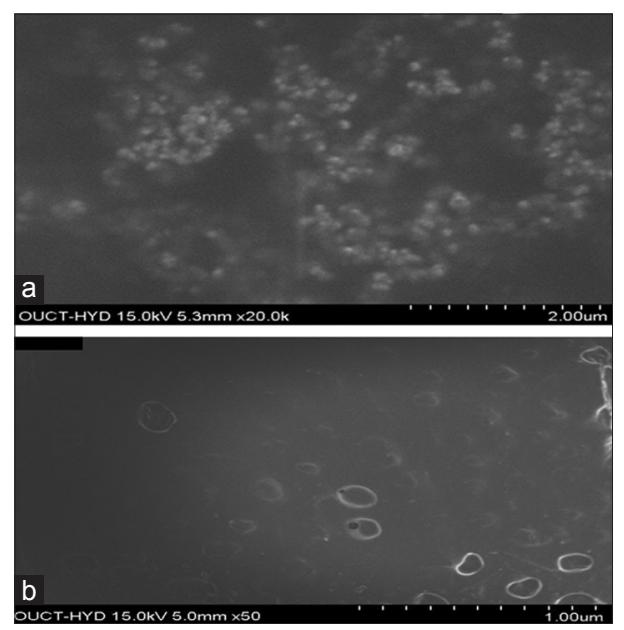

Fig. 16: ( $a$ and $b$ ) Scanning electron microscopy images of manidipine optimized formulations (F14)

stability studies. The studies indicated no significant variation in drug release and the drug content, physical and emulsifying properties.

\section{CONCLUSION}

The present research is aimed at the formulation and characterization of manidipine SNEDDS. The solubility studies indicated maximum solubility of drug in Capmul MCM (oil), Transcutol HP (Surfactant), and Lutrol L300 (cosurfactant). A tertiary phase diagram plotted in accordance with solubility studies indicates that self-emulsifying region was enhanced with increase in the concentration of surfactant and cosurfactant with oil. The visual observation tests indicate that the ratios $1: 9,2: 8,3: 7,4: 6$, and $5: 5$ of $S_{\text {mix }} 1: 1,7: 3,8: 2,9: 1,1: 9$, and $2: 8$ of $S_{\text {mix }} 2: 1$, and $5: 5,6: 4,7: 3,8: 2$, and $9: 1$ of $S_{\text {mix }} 3: 1$ showed rapid formation of emulsion within a minute having a clear appearance. Based on the results, fifteen manidipine liquid SNEDDS formulations F1-F15 were prepared and analyzed. The thermodynamic stability studies of all formulations indicate no change in visual description of samples. The \% transmittance study indicates that manidipine liquid SNEDDS formulation F14 has a value $>99 \%$ indicating high clarity of emulsion. The drug content of all formulations ranges from 91.99 to 98.96\% with maximum drug content observed in F14 and the maximum drug release found in F14. Hence, F14 is chosen as optimized liquid SNEDDS formulation of manidipine. Drug-excipient studies through FTIR indicated no interaction between manidipine and formulation excipients used. The particle size of F14 is $22.4 \mathrm{~nm}$, PI 0.313 , and Zeta potential $-5.1 \mathrm{mv}$. The SEM studies of optimized formulation F14 indicated spherical shape with uniform particle distribution. The formulation F14 subjected to stability studies for 6 months indicated no significant change in drug content, drug release, emulsifying properties, and appearance. Hence, a potential liquid SNEDDs formulation of manidipine developed with enhanced solubility, dissolution rate, and bioavailability.

\section{AUTHORS' CONTRIBUTION}

Two authors contributed equally.

\section{CONFLICTS OF INTEREST}

There are no conflicts of interest by authors.

\section{REFERENCES}

1. Charman WN, Stella VJ. Transport of lipophilic molecules by the intestinal lymphatic system. Adv Drug Deliv Rev 1991;7:1-14.

2. O'Driscoll CM. Lipid-based formulations for intestinal lymphatic delivery. Eur J Pharm Sci 2002;15:405-15.

3. Porter CJ, Trevaskis NL, Charman WN. Lipids and lipid-based formulations: Optimizing the oral delivery of lipophilic drugs. Nat Rev Drug Discov 2007;6:231-48.

4. Shafiq S, Shakeel F, Talegaonkar S, Ahmad FJ, Khar RK, Ali M, et al. Development and bioavailability assessment of ramipril nanoemulsion formulation. Eur J Pharm Biopharm 2007;66:227-43.

5. Attivi D, Ajana I, Astier A, Demoré B, Gibaud S. Development of microemulsion of mitotane for improvement of oral bioavailability. Drug Dev Ind Pharm 2010;36:421-7.

6. Dixit AR, Rajput SJ, Patel SG. Preparation and bioavailability assessment of SMEDDS containing valsartan. AAPS PharmSciTech 2010;11:314-21.

7. Nielsen FS, Petersen KB, Müllertz A. Bioavailability of probucol from lipid and surfactant based formulations in minipigs: Influence of droplet size and dietary state. Eur J Pharm Biopharm 2008;69:553-62.

8. Wu X, Xu J, Huang X, Wen C. Self-microemulsifying drug delivery system improves curcumin dissolution and bioavailability. Drug Dev Ind Pharm 2011;37:15-23.

9. Cui J, Yu B, Zhao Y, Zhu W, Li H, Lou H, et al. Enhancement of oral absorption of curcumin by self-microemulsifying drug delivery systems. Int J Pharm 2009;371:148-55.

10. McKeage K, Scott LJ. Manidipine: A review of its use in the management of hypertension. Drugs 2004;64:1923-40.

11. Cheer SM, McClellan K. Manidipine: A review of its use in hypertension. Drugs 2001;61:1777-99.

12. Chaudhari P, Bhalerao A. Method validation for spectrophotometric estimation of cilnidipine. Int J Pharm Pharm Sci 2012;4:96-98.

13. Talegaonkar S, Mustafa G, Akhter S, Iqbal ZI. Design and development of oral oil-in-water nanoemulsion formulation bearing atorvastatin: In vitro assessment. J Dispers Sci Technol 2010;31:690-701.

14. Setthacheewakul S, Mahattanadul S, Phadoongsombut N, Pichayakorn W, Wiwattanaptapee R. Development and characterization of solid self-emulsifying drug delivery system of cilnidipine. Eur J Pharm Biopharm 2010;76:475-85.

15. Constantinides PP, Scalart JP, Lancaster C, Marcello J, Marks G, Ellens $\mathrm{H}$, et al. Formulation and intestinal absorption enhancement 
evaluation of water-in-oil microemulsions incorporating medium-chain glycerides. Pharm Res 1994;11:1385-90.

16. Rajinikanth PS, Keat NW, Garg S. Self-nanoemulsifying drug delivery systems of valsartan: Preparation and in-vitro characterization. Int $\mathrm{J}$ Drug Deliv 2012;4:153-63.

17. Shafiq S, Shakeel F, Talegaonkar S, Ahmad FJ, Khar RK, Ali M, et al. Development and bioavailability assessment of ramipril nanoemulsion formulation. Eur J Pharm Biopharm 2007;66:227-43.

18. Costa P, Sousa Lobo JM. Modeling and comparison of dissolution profiles. Eur J Pharm Sci 2001;13:123-33.

19. Dixit P, Jain DK, Dumbwani J. Standardization of an ex vivo method for determination of intestinal permeability of drugs using everted rat intestine apparatus. J Pharmacol Toxicol Methods 2012;65:13-7.

20. Shaji J, Jadhav D. Newer approches to self emulsifying drug delivery system. Int J Pharm Pharm Sci 2010;2:37-42.

21. Surendra S, Roshan I, Nagori B. Formulation and evaluation of self-emulsifying drug delivery system of etoricoxib. Asian J Pharm Clin Res 2017;10:367-72. 